\title{
Quantificação de colágeno tipos I e III em lesões de pele de pacientes portadores de hanseníase
}

\section{Quantification of collagen types I and III in skin lesions of leprosy patients}

Igor Costa de Lima' (D), Shaumin Vasconcelos Wu² ${ }^{\mathbb{D}}$, Luciana Mota Silva ${ }^{3}$, Jofre Jacob da Silva Freitas ${ }^{4}$, Francisca Regina Oliveira Carneiro ${ }^{5}$, Fabíola Raquel Tenório Oliveira ${ }^{4}$ (D)

' Universidade do Estado do Pará, Centro de Ciências Biológicas e da Saúde, Departamento de Morfologia e Ciências Fisiológicas, Belém, Pará, Brasil 2 Universidade Federal do Pará, Belém, Pará, Brasil

${ }^{3}$ Centro Universitário do Pará, Belém, Pará, Brasil

${ }^{4}$ Universidade do Estado do Pará, Centro de Ciências Biológicas e da Saúde, Departamento de Morfologia e Ciências Fisiológicas, Laboratório de Morfofisiologia Aplicada à Saúde, Belém, Pará, Brasil

${ }^{5}$ Universidade do Estado do Pará, Centro de Ciências Biológicas e da Saúde, Ambulatório de Dermatologia, Belém, Pará, Brasil

\section{RESUMO}

OBJETIVO: Quantificar as fibras de colágeno tipos I e III nas formas clínicas polares e inicial da hanseníase. MATERIAIS E MÉTODOS: Foram analisadas 39 amostras de pele conservadas em blocos de parafina, distribuídas nos grupos: indeterminado - MHI (9), tuberculoide - MHT (16) e virchowiano - MHV (14) e submetidas à microtomia e coloração de Picrosirius Red. As imagens foram analisadas pelo programa Image J e, para a análise estatística, usou-se o teste ANOVA ( $p<0,05)$. RESULTADOS: O colágeno tipo I ocupou, em MHI, MHT e MHV, respectivamente, 69\% (DP $\pm 14,94), 54 \%$ $(\mathrm{DP} \pm 18,66)$ e $44 \%(\mathrm{DP} \pm 18,06)$ da área da derme, com diferença estatística significante entre $\mathrm{MHI}$ e $\mathrm{MHV}(\mathrm{p}<0,01)$. O colágeno tipo III ocupou, em MHI, MHT e MHV, respectivamente, 1,6\% (DP $\pm 1,524), 2,6 \%$ (DP $\pm 1,787)$ e 4,5\% $(\mathrm{DP} \pm 2,325)$, com diferenças estatísticas significantes entre MHI e MHV $(p<0,01)$ e entre MHV e MHT $(p<0,05)$. Quanto à correlação de colágeno tipos I e III, obteve-se na forma MHI uma correlação negativa, forte e com significância estatística $(r=-0,7000 ; p=0,0433)$; para $M H T$, obteve-se uma correlação negativa, moderada e com significância estatística ( $r=-0,5029 ; p=0,0471)$; enquanto para MHV não houve significância estatística $(p=0,7253 ; r=-0,1033)$. CONCLUSÃO: O tipo de hanseníase e seus aspectos imunopatológicos associados são determinantes nos níveis de colágeno identificados, observando-se gradualmente perda do colágeno tipo I e aumento do colágeno tipo III em MHI, MHV e MHT.

Palavras-chave: Hanseníase; Colágeno Tipo I; Colágeno Tipo III.

\begin{abstract}
OBJECTIVE: To quantify collagen fibers types I and III in the polar and initial clinical forms of leprosy. MATERIALS AND METHODS: A total of 39 skin samples preserved in paraffin blocks were analyzed, divided into undetermined - UL (9), tuberculoid - TL (16), and virchowian - VL (14), and submitted to Picrosirius Red microtomy and staining. The images were analyzed using the Image J software, and the ANOVA test was used for statistical analysis ( $<0.05)$. RESULTS: Type I collagen covered $69 \%$ (SD \pm 14.94$), 54 \%$ (SD + 18.66), and 44\% (SD \pm 18.06 ) of the dermis area in $U L, T L$, and $V L$, respectively, with a statistically significant difference between $\mathrm{UL}$ and $\mathrm{VL}(\mathrm{p}<0.01)$. Type III collagen covered $1.6 \%$ (SD \pm 1.524$)$, $2.6 \%$ (SD \pm 1.787 ), and $4.5 \%$ ( $S D \pm 2.325)$, of the dermis area in $U L, T L$, and $V L$, respectively, with statistically significant differences between UL and VL $(p<0.01)$ and between VL and TL $(p<0.05)$. As for the correlation of collagen types I and III, a negative, strong, and statistically significant correlation was obtained in the UL form ( $r=-0.7000 ; p=0.0433)$; for TL, a negative, moderate, and statistically significant correlation was obtained $(r=-0.5029 ; p=0.0471)$; while for $V L$ there was no statistical significance ( $p=0.7253 ; r=-0.1033)$. CONCLUSION: The type of leprosy and its associated immunopathological aspects are determinant in the collagen levels were identified, and gradually showing loss of type I collagen and increase of type III collagen in UL, VL and TL.
\end{abstract}

Keywords: Leprosy; Type I Collagen; Type III Collagen.

\section{Correspondência / Correspondence:}

Igor Costa de Lima

Trav. Perebebuí, 2623. Bairro: Marco. CEP: $66087-662$ - Belém, Pará, Brasil - Tel.: +55 (91) 98259-2947

E-mail: igorcstlm@gmail.com 


\section{INTRODUÇÃO}

O elevado poder de incapacitar a pessoa infectada faz com que a hanseníase seja caracterizada como um problema de saúde pública. Segundo dados do Ministério da Saúde1, em 2018, foram notificados 28.660 casos de hanseníase no Brasil, distribuindo-se nas regiões da seguinte maneira: Nordeste (40,9\%), Centro-Oeste (23,2\%), Norte (20,2\%), Sudeste (12,9\%) e Sul $(2,8 \%)$.

A hanseníase é uma infecção granulomatosa, crônica e degenerativa causada pelo Mycobacterium leprae, que afeta principalmente os nervos periféricos e a pele. Cerca de $95 \%$ das pessoas expostas ao bacilo do $M$. leprae são resistentes à infecção, sendo os $5 \%$ suscetíveis $^{2}$. Nos casos suscetíveis, as diversas formas em que a doença se manifesta resultam em distintas classificações, sendo a de Ridley e Jopling ${ }^{3}$ a principal, na qual leva-se em consideração alterações histopatológicas associadas a alterações imunológicas ${ }^{3}$. Evidenciou-se ainda, em alguns estudos, que somente a exposição ao $M$. leprae não é suficiente para o desenvolvimento da doença, estando envolvidos diversos fatores genéticos responsáveis pela suscetibilidade à hanseníase e ao seu amplo espectro de manifestações ${ }^{4}$.

A manifestação inicial da doença caracteriza-se geralmente pela forma indeterminada (MHI), um estado transitório que pode ser encontrado em indivíduos, cuja resposta imunológica ao bacilo não foi definida, geralmente em crianças. Essa forma pode, em seguida, evoluir para a cura espontânea ou para as demais manifestações clínicas 5 .

Por se tratar de uma doença espectral, a resposta imunológica transita entre os polos extremos. Na forma tuberculoide (MHT), evidencia-se o polo de resistência, marcado por uma intensa resposta celular mediada por linfócitos T auxiliadores de perfil 1 (Th1) que são responsáveis pela produção de uma série de citocinas pró-inflamatórias, como o fator de necrose tumoral (TNF- $\alpha$ ), estimulando o recrutamento de linfócitos e monócitos para o local da infecção. Esse perfil de resposta tem influência na proliferação bacilar e, consequentemente, nas características histopatológicas da MHT - formação de granulomas ${ }^{6,7}$.

Enquanto isso, o polo virchowiano (MHV) caracteriza-se pela suscetibilidade ao bacilo, sendo a resposta imunológica mediada por linfócitos de perfil 2 (Th2), associados a citocinas imunossupressoras, como o fator de crescimento transformador beta (TGF- $\beta$ ), responsáveis pelo desenvolvimento e reparo tecidual, controle, diferenciação e proliferação celular. Esse perfil de resposta contribui para a proliferação de linfócitos $B$ e secreção de anticorpos que não são eficazes na destruição do bacilo, resultando em grandes alterações histopatológicas no local da lesão, evidenciado pela presença de um infiltrado inflamatório denso com macrófagos parasitados pelo bacilo ${ }^{6,7}$.

Um dos aspectos mais relevantes da hanseníase é a lesão neural, resultado do processo inflamatório na tentativa de destruir o bacilo. Uma das principais causas de neuropatia na hanseníase é a fibrose, sendo que na MHV ocorre desmielinização e perda axonal, além de uma densa fibrose endoneural e perineural. Na MHT, os nervos estão total ou parcialmente destruídos e substituídos por tecido fibroso, enquanto que, na $\mathrm{MHI}$, há um número mínimo de infiltrado nos nervos ${ }^{8}$.

processo inflamatório é responsável por grandes alterações dos componentes da matriz extracelular (MEC) - colágeno, proteoglicanos, fibras elásticas e fibronectina - associados ao tipo de resposta imunológica em cada tipo de hanseníase, contribuindo para atrofia, degeneração tardia e, consequentemente, alterações biológicas das funções da pele na hanseníase?

Sendo os colágenos tipo I e III os mais abundantes na derme, o seu estudo na hanseníase é de grande relevância para a comunidade científica.

O colágeno tipo I é o mais comum e representa $90 \%$ do total de colágenos nos mamíferos, sendo sintetizado por fibroblastos, odontoblastos e osteoblastos, conferindo resistência às estruturas. $O$ TGF- $\beta$ e o fator de crescimento derivado de plaquetas estimulam a produção do colágeno tipo I por meio dos fibroblastos; em contrapartida, os glicocorticoides inibem a sua síntese $^{7,10}$. Já o colágeno tipo III é sintetizado pelos fibroblastos, pelos adipócitos e pelas células de Schwann (no sistema nervoso periférico), proporcionando fibras curtas e finas que normalmente são encontradas em tecidos que possuem um certo grau de elasticidade, como pele, músculos, fáscias e ligamentos. Além disso, é encontrado nas fases iniciais de cicatrização associado ao colágeno tipo I durante a fibrose e a fibrogênese ${ }^{10}$.

Logo, a avaliação de colágeno em amostras de pele de pacientes portadores de hanseníase é relevante, por essa doença manifestar-se principalmente sob a forma de lesões dérmicas e pelo colágeno ser um importante componente da MEC da derme, responsável pela manutenção do arcabouço normal. Dessa forma, o presente estudo visou quantificar e estabelecer uma correlação entre as fibras de colágeno tipos I e III nas formas clínicas polares (MHT e MHV) e inicial (MHI) da hanseníase.

\section{MATERIAIS E MÉTODOS}

\section{SELEÇÃO DAS AMOSTRAS}

Foram selecionadas 39 amostras de pele de pacientes conservadas em blocos de parafina do arquivo de patologia do Serviço de Dermatologia da Universidade do Estado do Pará, com diagnóstico clínico confirmado para hanseníase e avaliação histopatológica realizada, descritas nos laudos como: lesões de pele de MHI (nove), cuja derme apresentava infiltrado linfo-histiocitário perivascular e perineural sem formar granulomas (nos cortes corados pelo Fite-Faraco não se observou BAAR); lesões de pele de MHT (16), que se caracterizaram pela presença de granulomas epitelioides com células gigantes multinucleadas do tipo Langhans envolvendo vasos, anexos cutâneos e filetes nervosos dérmicos los cortes corados pelo Fite-Faraco 
não revelam BAAR); e lesões de pele de MHV (14), nas quais os cortes caracterizaram-se por uma epiderme delgada, com retificação papilar e faixa de Unna subepidérmica. $\mathrm{Na}$ derme, observou-se abundante infiltrado inflamatório formado por histiócitos com amplos citoplasmas claros, por vezes dilatados, repletos de BAAR evidenciados, formando grandes globias em cortes corados pelo Fite-Faraco. $O$ infiltrado apresentou disposição difusa, raramente esboçando granulomas malformados e se estendeu pela derme e pelo segmento hipodérmico representado, notando-se infiltração perineural.

As amostras de pele selecionadas pertenciam a pacientes maiores de 18 anos de idade, de ambos os sexos, que não haviam iniciado a poliquimioterapia no momento da biópsia.

Este trabalho foi aprovado pelo Comitê de Ética em Pesquisa da Universidade do Estado do Pará, Centro de Ciências Biológicas e da Saúde, em 18 de novembro de 2016, sob parecer $n^{\circ} 1.827 .246$.

\section{PROCESSAMENTO HISTOLÓGICO DAS AMOSTRAS}

Foram produzidos cortes com a espessura de $5 \mu \mathrm{m}$ (Micrótomo Leica modelo RM2255) e, posteriormente, corados com Picrosirius Red (Direct Red 80 Sigma-Aldrich, Saint Louis, EUA) para a observação das fibras de colágeno tipos I e III.

\section{ANÁLISE DAS AMOSTRAS E QUANTIFICAÇÃO DE COLÁGENO}

Os cortes histológicos foram observados em microscópio óptico sob luz polarizada. Esse método permitiu observar a diferenciação dos colágenos tipo I (birrefringência laranja-amarelada a laranja e vermelha) e tipo III (birrefringência verde ou verde-amarelada).

Para a captura das imagens, foi utilizada uma câmera de alta resolução (AxioCam MRc, Zeiss), acoplada ao microscópio Axio Scope.Al Zeiss e a um computador com o sistema operacional Microsoft Windows, contendo o programa AxioVision 4.2 (Zeiss). De cada lâmina, foram selecionados e fotomicrografados todos os campos da derme em aumento de 100x. As imagens obtidas foram analisadas com o auxílio do software Image J, com o plugin Threshold Colour, obtendo-se a porcentagem de colágeno, por meio da análise de partículas automatizadas pela seleção e medida das áreas com base na cor. $\bigcirc$ método padronizado por Bedoya et al. ${ }^{11}$, utilizado neste trabalho para a quantificação da área de colágeno, por meio do Threshold Colour, deu-se nos seguintes valores: matiz 0-40 para a cor vermelha (colágeno tipo I) e 45-120 para a cor verde (colágeno tipo (II), saturação 0-255 e brilho 5-225 para ambos os tipos de colágeno. Após a análise de todos os campos, realizou-se a média dos mesmos, obtendo-se a média de cada amostra dentro dos grupos.

\section{ANÁLISE ESTATÍSTICA}

Para a análise das medidas de tendência central, foi utilizado o teste ANOVA e o pós-teste Tukey, e Correlação de Spearman para identificar o tipo de relação entre os colágenos tipo I e III. Utilizou-se o nível de significância de $5 \%(p \leq 0,05)$.

\section{RESULTADOS}

Foram observadas alterações nos níveis de colágeno tipos I e III de forma padronizada ao analisar as micrografias de pele de pacientes portadores de $\mathrm{MHI}$, MHT e MHV. A análise, por meio do programa Image J, possibilitou destacar e quantificar as áreas relacionadas aos colágenos nos três tipos de hanseníase estudados. Para exemplificar essas afirmações, a figura 1 apresenta as micrografias originais obtidas sob luz polarizada, evidenciando os colágenos tipo I e III, e suas análises para identificação e quantificação de colágenos para $\mathrm{MHI}(\mathrm{A}, \mathrm{D}$ e $\mathrm{G}), \mathrm{MHT}(\mathrm{B}, \mathrm{E}$ e H) e $\mathrm{MHV}$ (C, $\mathrm{F}$ e I). Os valores foram expressos em porcentagem de área. Observa-se, na figura 1, que entre as formas $\mathrm{MHI}, \mathrm{MHT}$ e $\mathrm{MHV}$, os níveis do colágeno tipo I reduziram enquanto, para o colágeno tipo III, os níveis aumentaram.

Nas lesões de $\mathrm{MHI}$, o colágeno tipo I ocupou em média 69\% da área da derme (DP $\pm 14,94)$ e o colágeno tipo III, 1,6\% (DP $\pm 1,524)$. Nas lesões de MHT, o colágeno tipo I ocupou em média 54\% da área da derme (DP $\pm 18,66)$ e o colágeno tipo III, $2,6 \%$ (DP $\pm 1,787)$. Já nas lesões de $M H V$, a média do colágeno tipo I foi de 44\% (DP $\pm 18,06)$ e do colágeno tipo III foi de 4,5\% (DP $\pm 2,325)$. Entretanto, observou-se uma tendência de maior presença do colágeno tipo I nas lesões $\mathrm{MHI}$ (69\%), seguidas das MHT (54\%) e MHV (44\%), com diferença estatística significante entre $\mathrm{MHI}$ e MHV $(\mathrm{p}<0,01)$. Por outro lado, o colágeno tipo III foi mais predominante nas lesões MHV (4,5\%), seguido das MHT $(2,6 \%)$ e $\mathrm{MHI}(1,6 \%)$, com diferenças estatísticas significantes entre $\mathrm{MHI}$ e MHV $(p<0,01)$ e entre MHT e MHV ( $\mathrm{p}<0,05)$ (Gráficos 1 e 2).

Quanto à correlação do colágeno tipos l e III nas formas estudadas, por meio do teste de Spearman, obteve-se, na forma MHI (Gráfico 3), uma correlação negativa, forte e com significância estatística ( $r=-0,7000 ; p=0,0433)$; na MHT (Gráfico 4), obteve-se uma correlação negativa, moderada e com significância estatística ( $r=-0,5029$; $p=0,0471$ ); enquanto, na MHV (Gráfico 5), não houve significância estatística para a correlação $(r=-0,1033$; $p=0,7253)$.

\section{DISCUSSÃO}

A análise dos componentes da matriz extracelular dérmica de lesões cutâneas de hanseníase feita por Antunes et al. ${ }^{9}$ revelou a remodelação da matriz extracelular dérmica compacta por uma matriz solta nas regiões ocupadas por granulomas. Uma matriz frouxa facilita a renovação celular promovida pela colagenase, elastase e deposição de fibronecina, que ocorreu mais intensamente na MHV em relação à MHT. Constatou-se ainda, por meio de imunomarcação, a substituição de fibras colágenas entrelaçadas densas por uma malha reticular, sendo observada, de forma mais intensa, no polo $\mathrm{MHV}$ ? 


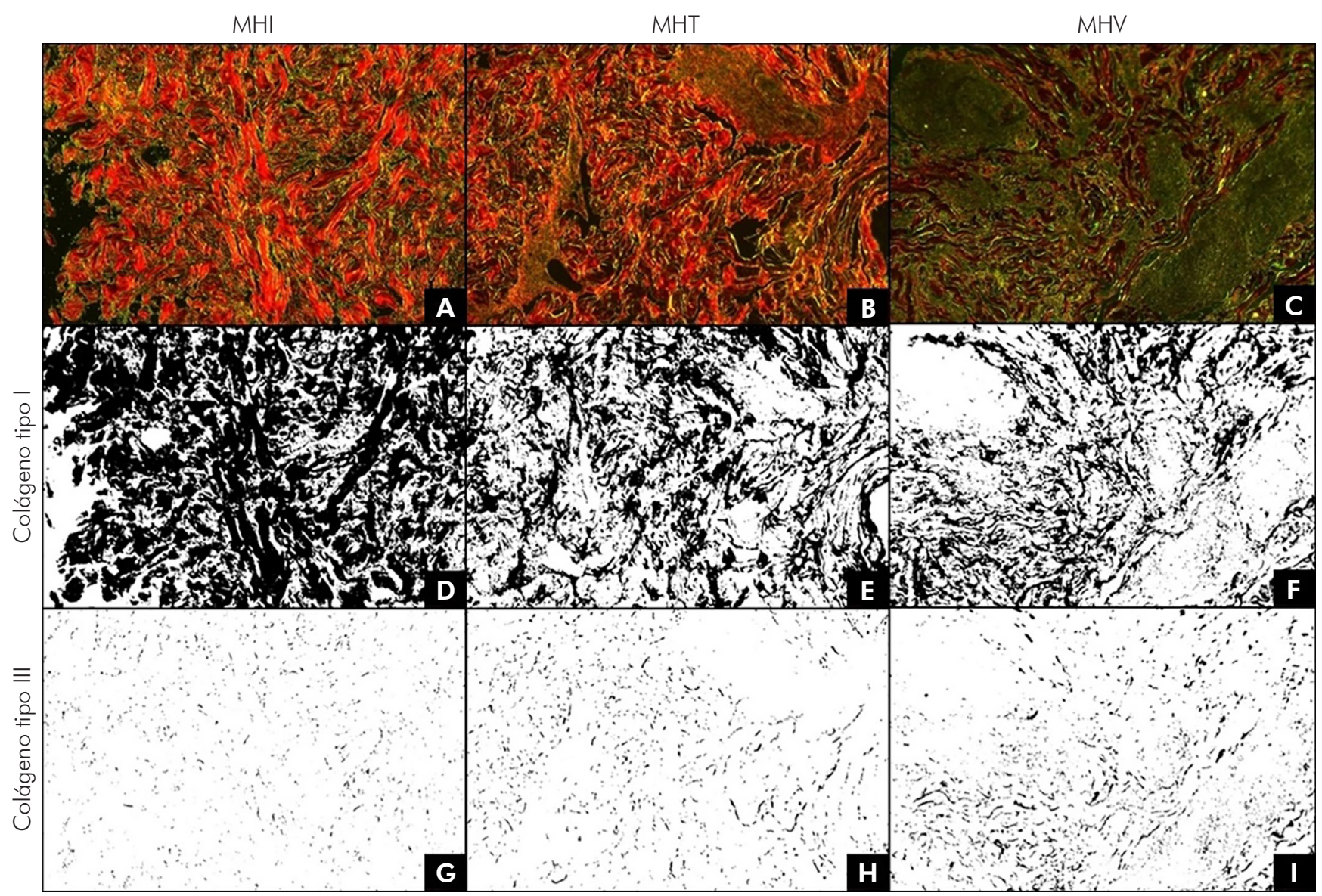

As imagens foram analisadas pelo programa Image J, para a seleção das áreas pertencentes aos colágenos tipo I e III. Em $\mathbf{D}$, tem-se a seleção da área correspondente ao colágeno tipo I $(61,5 \%)$ e, em $\mathbf{G}$, ao colágeno tipo III $(1,2 \%)$, em relação à micrografia $\mathbf{A}$; em $\mathbf{E}$, tem-se a seleção da área correspondente ao colágeno tipo I $(37,6 \%)$ e, em $\mathbf{H}$, ao colágeno tipo III $(2,6 \%)$, em relação à micrografia $\mathbf{B} ;$ e, em $\mathbf{F}$, tem-se a seleção da área correspondente ao colágeno tipo I (26,7\%) e, em I, ao colágeno tipo III (5,3\%), em relação à micrografia $\mathbf{C}$. Valores expressos em porcentagem de área.

Figura 1 - Micrografias em luz polarizada de amostra de pele de paciente com MHI (A), MHT (B) e MHV (C), evidenciando, em vermelho, o colágeno tipo I e, em verde, o colágeno tipo III (Picrosirius Red)

Colágeno tipo $\mid$

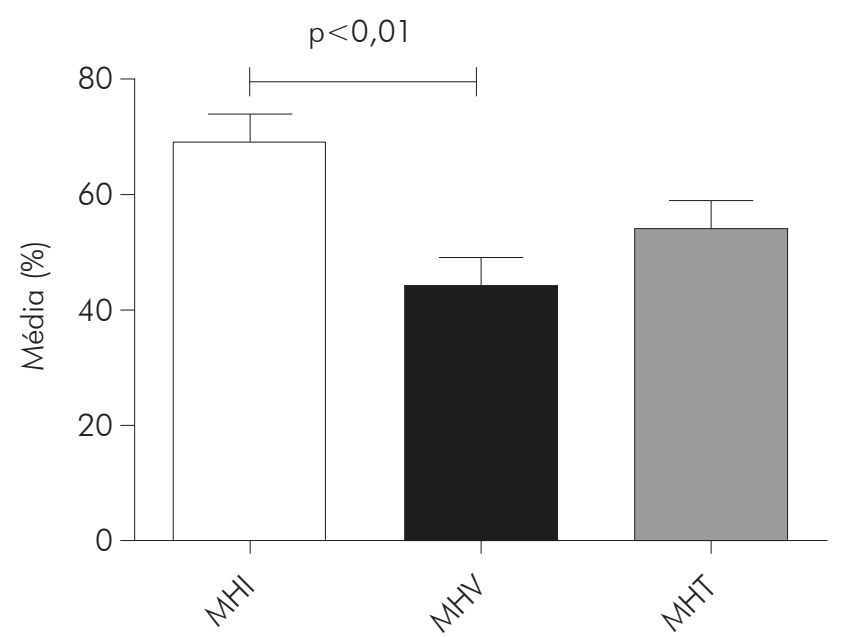

Gráfico 1 - Médias, expressas em porcentagem, da área do colágeno tipo I em lesões de pele de pacientes portadores de hanseníase nas formas $\mathrm{MHI}$ $(\mathrm{N}=14), \mathrm{MHV}(\mathrm{N}=9)$ e MHT $(\mathrm{N}=16)$, após a análise das imagens pelo programa Image $\mathrm{J}$ e a realização das médias por paciente e, em seguida, por grupo
Colágeno tipo III

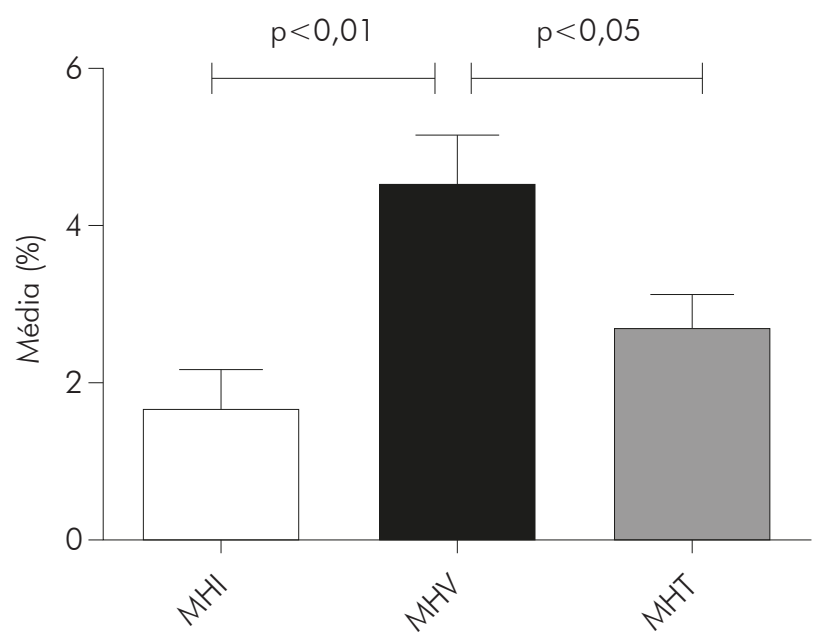

Gráfico 2 - Médias, expressas em porcentagem, da área do colágeno tipo III em lesões de pele de pacientes portadores de hanseníase nas formas $\mathrm{MHI}$ $(N=14), \operatorname{MHV}(N=9)$ e MHT $(N=16)$, após análise das imagens pelo programa Image J e realização das médias por paciente e, em seguida, por grupo 


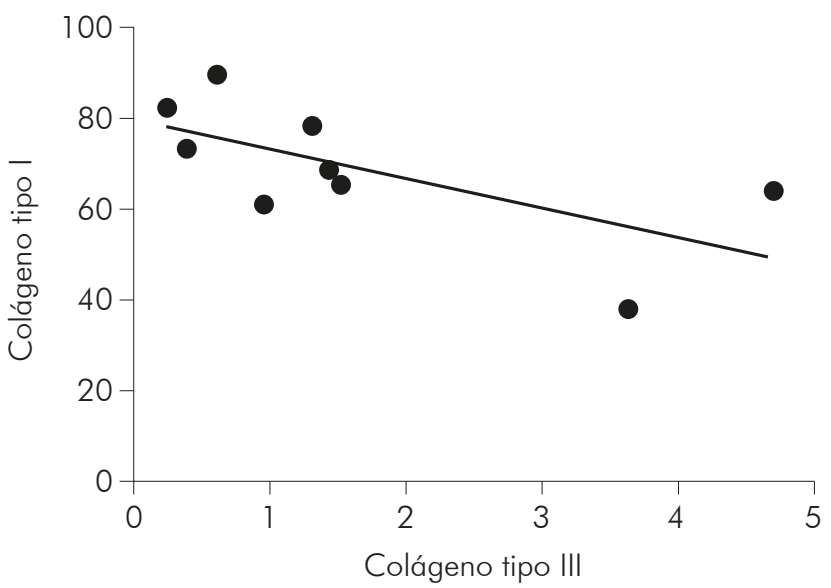

Gráfico 3 - Correlação entre colágenos I e III em MHI $(r=-0,7000 ; p=0,0433)$

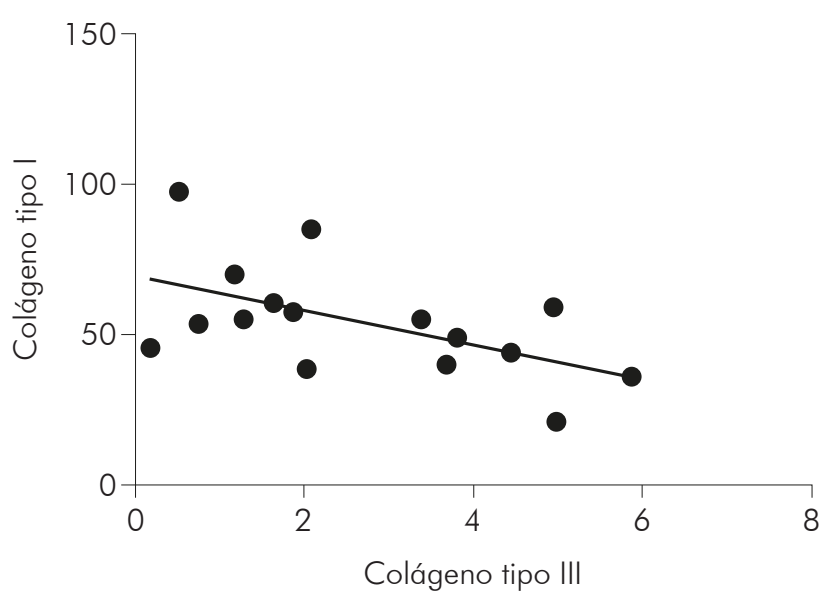

Gráfico 4 - Correlação entre colágenos I e III em MHT $(r=-0,5029 ; p=0,0471)$

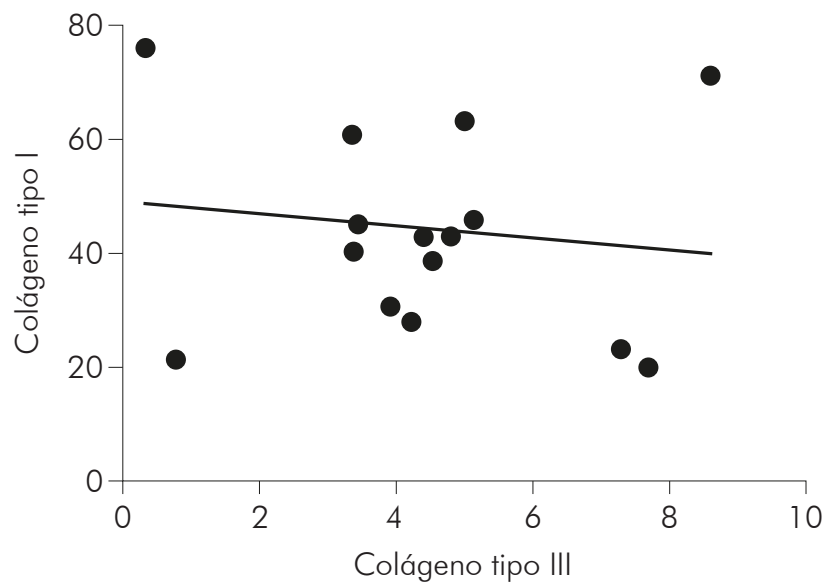

Gráfico 5 - Correlação entre colágenos I e III em MHV $(r=-0,1033 ; p=0,7253)$
Durante o processo inflamatório, estruturas vasculares, epiteliais e componentes da MEC são essenciais, estimulando a migração de leucócitos, secretando citocinas estimulatórias ou funcionando como substrato de adesão. A troca da matriz extracelular densa por uma matriz frouxa fornece - ambiente ideal para a atuação de mediadores e citocinas do processo inflamatório. A substituição da MEC ocorre principalmente por colagenólise, degradação de proteoglicanos, elastólise e neosíntese de colágeno, influenciadas por fatores como colagenase e elastase produzida por macrófagos, encarregados de remover os componentes originais da matriz extracelular, deposição de fibronectina e produção de fibroblastos, que são responsáveis por criar um novo substrato essencial à invasão de células mononucleares e à produção de colágeno estimulada por citocinas fibrogênicas ${ }^{12}$.

Portanto, o equilíbrio de citocinas fibrogênicas e fibrolíticas é responsável pelos níveis de colágeno identificados em lesões inflamatórias. Assim, os moduladores imunológicos podem influenciar nos níveis de colágeno observados durante o processo inflamatório causado pelo bacilo.

Sob essa perspectiva, sabe-se que a interleucina-1 (IL-1) é um importante modulador estimulante da fibrogênese, enquanto o interferon-gama (IFN-y) é um inibidor desse processo ${ }^{13}$. $\mathrm{Na} \mathrm{MHV}$, há uma diminuição de IFN-y ${ }^{13}$, podendo-se associar, de forma especulativa, que a alteração nos níveis de colágeno observados neste trabalho foram influenciados pela ação dessa enzima.

$\bigcirc$ TGF- $\beta$ é um fundamental estimulador do desencadeamento da produção do colágeno tipos I e III, estimulando a produção de RNAm das metaloproteinases da matriz e do fator de crescimento do nervo nas células de Schwann. Dentre os eventos destacados para a ação dessa citocina, destacam-se a reprogramação do fenótipo das células, influenciando no processo de fibrogênese, e a evolução das lesões dos nervos e na apoptose durante o progresso da doença $^{7,14}$.

Estudos recentes mostraram a participação de novos tipos de linfócitos Th produtores de mediadores que intensificam a resposta inflamatória na forma resistente da doença, como as células CD4+ e Th17, que produzem citocinas, como a interleucina 17 (IL-17), responsáveis por recrutar monócitos e neutrófilos para o local da infecção ${ }^{15}$.

A IL-17 induz uma lesão nervosa, estimulando uma resposta inflamatória visando a manutenção da matriz extracelular. Além disso, a IL-17 regula negativamente a produção do fator de crescimento nervoso (NGF) e do receptor de NGF (NGF R) ${ }^{15,16}$.

estudo de Aarão et al. ${ }^{17}$ revelou uma elevada expressão de IL-17 na MHT, responsável por desencadear um processo inflamatório para a 
manutenção da matriz dérmica. Além disso, mostrou uma alta expressão de NGF e NGF R na MHV, que são responsáveis por processos como regeneração de nervos da pele e estimulação de fibroblastos que induzem o processo de cicatrização, uma vez que em MHV há elevado grau de degradação da matriz dérmica acompanhado de baixa resposta imunológica. Assim, pode-se sugerir que o colágeno tipo III elevado na MHV está sendo influenciado pela presença desses mediadores ${ }^{17}$.

Dentre as formas da hanseníase utilizadas neste estudo (MHI, MHT e MHV), destacam-se, como característica histológica de cada uma, respectivamente: presença de infiltrado discreto, a formação de granulomas, sendo responsáveis por uma forte resposta imune, e a presença de infiltrado inflamatório difuso, com macrófagos parasitados por bacilos e globias na $\mathrm{MHV}^{6}$. Ao comparar as características histopatológicas da hanseníase identificadas em outras patologias, também foram observadas alterações nos níveis de colágeno ${ }^{18,19,20}$.

O trabalho de Silva-Almeida et al. ${ }^{18}$ com ratos infectados por Leishmania amazonensis demonstrou que, em regiões onde havia um intenso infiltrado inflamatório, houve maior produção do colágeno tipo III, enquanto que em outras regiões, onde o processo inflamatório era mais discreto, os níveis de colágeno se aproximaram dos valores do grupo controle. Observou-se ainda que, nos ratos infectados, houve alterações da estrutura normal da derme, caracterizadas pela destruição do colágeno tipo I. Esses resultados reforçam os achados desta pesquisa, uma vez que, em amostras de alterações histopatológicas intensas (MHV), foram encontrados maiores níveis do colágeno tipo III em relação a amostras cujas alterações principais eram de granulomas (MHT) e infiltrado inflamatório discreto (MHI).

Pesquisas feitas por Abreu-Silva et al. ${ }^{19}$, em infecções experimentais por L. amazonensis em pele de camundongos, mostraram a substituição do colágeno tipo I pelo tipo III no decorrer da infecção. Esse aumento do colágeno tipo III também foi identificado nos trabalhos de Gonçalves et al. ${ }^{20}$ em cães infectados por Leishmania chagasi com pneumonite intersticial crônica. A presença das fibras reticulares, nesse caso, foi frequentemente identificada em áreas de infiltrado inflamatório intenso.

Os resultados encontrados na literatura coincidem com os achados do presente estudo, uma vez que foram identificados a diminuição do colágeno tipo I e o aumento do colágeno tipo III em um processo de lesão crônica, estabelecendo-se ainda uma correlação de ambos os tipos de colágeno com significância estatística, mesma relação encontrada em processos de reparação tecidual.

Tais resultados estão em consonância com os achados deste estudo, uma vez que se identificou uma maior produção do colágeno tipo III nas lesões de pacientes do grupo MHV, seguidos pelos MHT e MHI. Sabe-se que na MHV o processo inflamatório é mais intenso do que na MHT6.

Acredita-se que a prevalência de infiltrado inflamatório inespecífico na $\mathrm{MHI}$, de granulomas nodulares na MHT e de infiltrados inflamatórios difusos na $\mathrm{MHV}^{6}$ determinam, nessa ordem, níveis crescentes de gravidade das manifestações clínicas da hanseníase, visto que se observam maior perda do colágeno tipo I e aumento dos níveis do colágeno tipo III, de acordo com a gravidade das manifestações clínicas/histopatológicas, como destacado na figura 1 em três micrografias, sendo cada uma referente a um tipo de hanseníase pesquisada neste estudo.

Observou-se, ainda, uma correlação negativa e forte entre os tipos de colágenos avaliados na $\mathrm{MHI}$ e negativa e moderada na MHV, indicando uma relação inversamente proporcional nos níveis de colágeno. Acredita-se que, devido ao dano à matriz extracelular causado pelo processo inflamatório da doença, os níveis do colágeno tipo III tenham aumentado em detrimento dos níveis do colágeno tipo I. Isso se deve à associação entre essas fibras, que são encontradas na derme, principalmente em processos de reparação tecidual ${ }^{21}$.

\section{CONCLUSÃO}

Assim, acredita-se que as lesões na fase inicial e polares da hanseníase apresentam diferenças na quantidade de fibras colágenas tipo I e III, em decorrência do processo inflamatório persistente, característico da doença, que ocorre em diferentes intensidades de acordo com a forma clínica da hanseníase observada. Tal diferença ocorre de forma gradual, de acordo com a gravidade da lesão provocada pelas diferentes manifestações da hanseníase, sendo a $\mathrm{MHI}$ a que provoca lesões menos graves, seguidas pela MHT e pela MHV.

Dentro dessa perspectiva, observou-se que os níveis de colágeno se encontraram nesses mesmos padrões, sendo que, enquanto o colágeno tipo I diminuiu progressivamente nas lesões de $\mathrm{MHI}, \mathrm{MHT}$ e MHV, o colágeno tipo III aumentou na mesma sequência.

A perda do colágeno tipo I provocada pela lesão persistente pode resultar na destruição do arcabouço normal da derme e substituição da matriz original densa por uma matriz frouxa, propícia ao processo de renovação tecidual, associada ao aumento dos níveis do colágeno tipo III observados em áreas de intenso infiltrado inflamatório.

Além disso, evidencia-se também que há uma correlação inversamente proporcional nos níveis de colágeno tipos I e III, considerados fortes e moderados para $\mathrm{MHI}$ e $\mathrm{MHT}$, respectivamente. Considera-se, até então, que o mesmo ocorre devido ao processo de remodelação da matriz extracelular provocado pela presença da lesão.

É importante ressaltar igualmente a necessidade de se avaliar, em pesquisas futuras, a influência dos diversos moduladores imunológicos na patogênese da 
hanseníase, bem como sua participação no processo inflamatório.

\section{AGRADECIMENTOS}

Ao Laboratório de Morfofisiologia Aplicada à Saúde da Universidade do Estado do Pará, pelos materiais, equipamentos e espaço necessários para o desenvolvimento desta pesquisa; e à Sirley Adriana Ortiz Bedoya, da Universidade Federal de Viçosa, pelo imenso auxílio e apoio com a metodologia de quantificação de colágeno.

\section{CONFLITOS DE INTERESSES}

Os autores declaram não haver conflitos de interesses em relação à pesquisa.

\section{CONTRIBUIÇÃO DOS AUTORES}

Todos os autores contribuíram com a idealização do estudo, a análise e a interpretação dos dados e com a redação do manuscrito, aprovando a versão final publicada. Declaram-se responsáveis pelo conteúdo integral do artigo, garantindo sua precisão e integridade.

\section{REFERÊNCIAS}

1 Ministério da Saúde (BR). Casos novos de hanseníase por estados e regiões, Brasil, 1990 a 2018 [Internet]. Brasília: Ministério da Saúde; 2019 [citado 2019 mai 23]. Disponível em: https:// www.saude.gov.br/images/pdf/2019/julho/17/ Casos-novos-de-hansen--ase-por-estados-e-regi-es--Brasil--1990-a-2018.pdf.

2 Ministério da Saúde (BR). Secretaria de Vigilância em Saúde. Hanseníase. Bol Epidemiol. 2018;49(4):1-10.

3 Ridley DS, Jopling WH. Classification of leprosy according to immunity: a five-group system. Int J Lepr Other Mycobact Dis. 1966;34(3):255-73.

4 Silvestre MPSA, Lima LNGC. Hanseníase: considerações sobre o desenvolvimento e contribuição (institucional) de instrumento diagnóstico para vigilância epidemiológica. Rev Pan-Amaz Saude. 2016;7(no. esp):93-8.

5 Ministério da Saúde (BR). Secretaria de Vigilância em Saúde. Departamento de Vigilância e Doenças Transmissíveis. Guia prático sobre a hanseníase. Brasília: Ministério da Saúde; 2017.

6 Obadia DL, Verardino G, Alves MFGS. Hanseníase: correlação clínico-histopatológica. Rev Hosp Univ Pedro Ernesto. 2011 mar;10(1):20-3.

7 Sousa JR, Silva PYA, Soares LPMA, Aarão TLS, Pinto DS, Fuzii HT, et al. Imunoexpressão de TNF- $\alpha$ e TGF- $\beta$ em lesões de pacientes nas diversas formas clínicas da hanseníase por meio da técnica de imunoistoquímica. Rev Pan-Amaz Saude. 2013 jun;4(2):45-53.

8 Chimelli L. Valor da biópsia de nervo no diagnóstico da hanseníase - auxílio à clínica nos casos de forma neural pura e reativação. Hansen Int Special. 1998:89-92.

9 Antunes SL, Gallo ME, Almeida SM, Mota E, Pelajo M, Lenzl HL. Dermal extracellular matrix in cutaneous leprosy lesions. Int J Lepr Other Mycobact Dis. 1999 Mar;67(1):24-35.
10 Calvi ENC, Nahas FX, Barbosa MV, Calil JA, Ihara SSM, Silva MS, et al. An experimental model for the study of collagen fibers in skeletal muscle. Acta Cir Bras. 2012 Oct;27(10):681-6.

11 Bedoya SAO, Conceição LG, Viloria MIV, Loures $\mathrm{FH}$, Valente FL, Amorim RL, et al. Caracterização de colágenos tipos I e III no estroma do carcinoma de células escamosas cutâneo em cães. Arq Bras Med Vet Zootec. 2016 jan-fev;68(1): 147-54.

12 Ferraz FB, Fernandez JH. Integrinas na adesão, migração e sinalização celular: associação com patologias e estudos clínicos. Rev Cient Fac Med Campos. $2014 ; 9(2): 25-34$

13 Nogueira N, Kaplan G, Levy E, Sarno EN, Kushner P, Granelli-Piperno A, et al. Defective gamma interferon production in leprosy. Reversal with antigen and interleukin 2. J Exp Med. 1983 Dec;158(6):2165-70.

14 Petito RB, Amadeu TP, Pascarelli BMO, Jardim MR, Vital RT, Antunes SL, et al. Transforming growth factor- $\beta$ may be a key mediator of the fibrogenic properties of neural cells in leprosy. J Neuropathol Exp Neurol. 2013 Apr;72(4):351-65.

15 Saini C, Ramesh V, Nath I. CD4+ Th17 cells discriminate clinical types and constitute a third subset of non Th1, Non Th2 T cells in human leprosy. PLoS Negl Trop Dis. 2013 Jul;7(7):e2338.

16 Stettner M, Lohmann B, Wolffram K, Weinberger JP, Dehmel T, Hartung HP, et al. Interleukin-17 impedes Schwann cell-mediated myelination. J Neuroinflammation. 2014;11:63.

17 Aarão TLS, Sousa JR, Botelho BS, Fuzii HT, Quaresma JAS. Correlation between nerve growth factor and tissue expression of $1 \mathrm{~L}-17$ in leprosy. Microb Pathog. 2016 Jan;90:64-8.

18 Silva-Almeida M, Carvalho LOP, Abreu-Silva AL, Souza CSF, Hardoim DJ, Calabrese KS. Extracellular matrix alterations in experimental Leishmania amazonensis infection in susceptible and resistant mice. Vet Res. 2012;43:10. 
19 Abreu-Silva AL, Calabrese KS, Mortara RA, Tedesco RC, Cardoso FO, Carvalho LOP, et al. Extracellular matrix alterations in experimental murine Leishmania (L.) amazonensis infection. Parasitology. 2004 Apr;128(4):385-90.

20 Gonçalves R, Tafuri WL, Melo MN, Raso P, Tafuri WL. Chronic interstitial pneumonitis in dogs naturally infected with Leishmania (Leishmania) chagasi: a histopathological and morphometric study. Rev Inst Med Trop S Paulo. 2003 May-Jun;45(3):153-8.
21 Tazima MFGS, Vicente YAMVA, Moriya T. Biologia da ferida e cicatrização. Medicina (Ribeirão Preto). 2008;41(3):259-64. 